\title{
The Polymorphisms rs2516839 of USF1 and -173G/C of MIF were not Associated with Coronary Artery Disease but Dyslipidemia in a Chinese Population
}

\author{
Chaoneng Wu ${ }^{1,2 \#}$, Yunguo Gong ${ }^{1,2 \#}$, Xiao Zhu ${ }^{3}$, Jie Yuan ${ }^{1,2}$, Aijun Sun ${ }^{1,2}$, Yunzeng Zou ${ }^{1,2 *}$ and Junbo Ge ${ }^{1,2 *}$ \\ ${ }^{1}$ Shanghai Institute of Cardiovascular Diseases, Zhongshan Hospital, Fudan University, Shanghai, China \\ 2Institute of Biomedical Science, Fudan University, China \\ ${ }^{3}$ Guangdong Province Key Laboratory of Medical Molecular Diagnosis, Guangdong Medical College, Dongguan, China \\ \#These authors contributed equally to the research
}

\begin{abstract}
Objective: The genome-wide association studies have pointed out lots of disease-associated variants in coronary artery disease (CAD). However, whether the polymorphisms of rs2516839 of upstream transcription factor (USF1) and -173G/C (rs755622) of macrophage migration inhibitory factor (MIF) are associated with CAD has remained undetermined. This study was to explore the associations between these two polymorphisms and the risk of CAD and dyslipidemia.
\end{abstract}

Methods: A case-control study was carried out in 654 angiographic confirmed CAD patients and 455 none-CAD control subjects. The polymorphisms were detected by TaqMan SNP Genotyping Assays.

Results: We did not observe significant association between the polymorphism of rs2516839 of USF1 with CAD risk, neither -173G/C (rs755622) of MIF. In the subgroup analysis of myocardial infarction and hypertension, the associations for theses two polymorphisms were negative also. However, the GG genotype carriers of rs2516839 of USF1 showed significantly lower levels of cholesterol, low-density lipoprotein cholesterol and apolipoprotein B.

Conclusions: Our findings showed that the rs 2516839 of USF1 and $-173 \mathrm{G} / \mathrm{C}$ (rs755622) of MIF do not contribute to CAD risk. Nevertheless, the rs2516839 of USF1 might have a protection for the dyslipidemia disorders.

Keywords: Polymorphism; Coronary artery disease; USF1; MIF; Dyslipidemia

\begin{abstract}
Abbreviations: MIF: Macrophage Migration Inhibitory Factor; USF1: Upstream Transcription Factor1; CAD: Coronary Artery Disease; IL: Interleukin; FCHL: Familial Combined Hyperlipidemia; BMI: Body Mass Index; Lp(a): Lipoprotein(a); ALL: Acute Lymphoblastic Leukemia; CHO: Cholesterol; LDL-C: Low-Density Lipoprotein Cholesterol; apoB: Apolipoprotein B
\end{abstract}

\section{Introduction}

Cardiovascular diseases are affected by both genetics and environmental factors [1]. The evidences that genetic factors contribute to the developing of cardiovascular diseases come from twin studies and familial aggregation. Further, the case-control genetic association studies have provided much useful information recently [2]. According to the results of the association studies, those genes involving in metabolic disorders as well as chronic inflammation process are considered to play important roles in CAD.

Macrophage migration inhibitory factor (MIF) is one of the critical genes for inflammation response. As a potent upstream regulator in the inflammatory process, MIF up regulates the inflammatory cytokines, including tumor necrosis factor- $\alpha$, interleukin (IL)-1 $\beta$, IL-6, IL-8, and prostaglandin E2 in response to lipopolysaccharide [3]. In addition, MIF has been reported to play a role in the development of unstable plaque, ischemia-reperfusion injury, as well as diabetes and obesity [4]. The expression of MIF can be affected by rs755622 (-173G/C), a polymorphism locating in the promoter region of MIF [5]. Even a series of studies have been reported the positive association between rs755622 of MIF and chronic inflammation, such as rheumatoid arthritis and adult-onset Still's disease, psoriasis, prostate cancer, childhood acute lymphoblastic leukemia (ALL), and obesity, no evidence can be found for the association between rs755622 of MIF and CAD [6-10].
Lipids disorder is a major risk factor for CAD development. Reports showed that upstream transcription factor (USF1) regulates lipid accumulation and dysfunction of USF1 contributes to familial combined hyperlipidemia (FCHL) [11]. As a ubiquitously expressed transcription factor, USF1 regulates more than 40 cardiovascular related genes. The polymorphism of rs 2516839 is localized in the non-coding region of the second exon of USF1. In Caucasians, this polymorphism was associated with increased risk for sudden cardiac death and triglyceride [12]. In another study in Hong Kong Chinese population, rs2516839 was positively associated with type 2 diabetes and/or metabolic syndrome [13]. Until now, the association of rs2516839 of USF1 with CAD in Chinese population has remained unclear.

Thus, the goal of the present study was to examine the association of rs755622 of MIF and rs2516839 of USF1 with CAD and lipids profile based on a Chinese population.

*Corresponding authors: Junbo Ge, Shanghai Institute of Cardiovascular Diseases, Zhongshan Hospital, Fudan University, 180 Feng Lin Road, Shanghai 200032, China, Tel: 86-21-64041990; Fax: 86-21-6422 3006; E-mail: ge.junbo2@zs-hospital.sh.cn

Yunzeng Zou, Shanghai Institute of Cardiovascular Diseases, Zhongshan Hospital, Fudan University, 180 Feng Lin Road, Shanghai 200032, China, Tel: 86-21 64041990; Fax: 86-21-6422 3006; E-mail: zou.yunzeng@zs-hospital.sh.cn

Received August 05, 2014; Accepted September 29, 2014; Published October 06, 2014

Citation: Wu C, Gong Y, Zhu X, Sun A, Yuan J, et al. (2014) The Polymorphisms rs2516839 of USF1 and $-173 \mathrm{G} / \mathrm{C}$ of MIF were not Associated with Coronary Artery Disease but Dyslipidemia in a Chinese Population. J Cardiovasc Dis Diagn 2: 177. doi:10.4172/2329-9517.1000177

Copyright: (c) 2014 Wu C, et al. This is an open-access article distributed under the terms of the Creative Commons Attribution License, which permits unrestricted use, distribution, and reproduction in any medium, provided the original author and source are credited. 
Citation: Wu C, Gong Y, Zhu X, Sun A, Yuan J, et al. (2014) The Polymorphisms rs2516839 of USF1 and -173G/C of MIF were not Associated with Coronary Artery Disease but Dyslipidemia in a Chinese Population. J Cardiovasc Dis Diagn 2: 177. doi:10.4172/2329-9517.1000177

Page 2 of 4

\section{Methods}

\section{Subjects}

Subjects were recruited from patients who underwent coronary angiography. Both the CAD and the control subjects had symptoms of chest discomfort. The CAD patients confirmed by angiography $(n=654)$ and the controls $(n=455)$ excluded by angiography were enrolled. The study was approved by the local research and ethics committee. Written informed consent was obtained from each participant before the percutaneous coronary intervention procedure. Blood was collected from the participants, and genomic DNA was isolated from leukocytes. Plasma lipids and glucose were measured by standard enzyme methods.

\section{Gene polymorphism analysis}

TaqMan MGB probes (FAMTM and VICR dye-labeled, Applied Biosystems, Foster City, Calif) polymerase chain reaction method was used for genotype determination. Reactions were performed with conditions: $10 \mathrm{~min}$ at $95^{\circ} \mathrm{C}$ and then 40 cycles of denaturation at $92^{\circ} \mathrm{C}$ for $15 \mathrm{~s}$ and annealing and extension at $60^{\circ} \mathrm{C}$ for $1 \mathrm{~min}$. After PCR, fluorescence of the VIC and of the FAM was measured by $7900 \mathrm{HT}$ genetic analyzer using SDS 1.2 software. We set four duplicates on each 384-well plate and the results of the duplicate samples agreed with each other.

\section{Statistical analysis}

The power of the study was evaluated by using "pssetup3.exe" http://biostat.mc.vanderbilt.edu/wiki/Main/PowerSampleSize. The Hardy-Weinberg equilibrium tests and the allele and genotype frequency analyses were conducted online on the SHESIS software platform (http://analysis.bio-X.cn/). The Bonferonni correction was used to correct $\mathrm{p}$ value, $1-(1-\alpha)^{\beta}$ for multiple comparisons. The Phi coefficient of Spearman correlation coefficients was calculated to assess the association between two continuous variables. $P$ value $<0.05$ was considered statistically significant.

\section{Results}

For the clinical characteristics, the CAD patients had larger percentage of male and hypertension (Table 1). For -173G/C of MIF and rs2516839 of USF1, we did not observe significant difference between $\mathrm{CAD}$ and the control group with regard to either the alleles or the genotypes $(P>0.05$, Table 2$)$. Then, the CAD cases were stratified by sex, age of onset, hypertension, and myocardial infarction (MI) for each stratum. We still failed to observe significant difference in all these subgroups analysis $(P>0.05$, data not shown). Further, even though CAD was significantly correlated with age, sex, smoking, hypertension, diabetes, and the levels of HDL-C and apoA (all $P<0.05$, Table 3 ), there was no correlation between the two polymorphisms and CAD (all $P>0.05$, Table 3).

Interestingly, when analysis with lipids profiles, we observed that, rs2516839 of USF1 was significantly associated with the levels of cholesterol $(P=0.01)$, LDL-C $(P=0.01)$, and apoB $(P=0.04)$. In addition, the levels of cholesterol, LDL-C and apoB were significant lower in subjects with GG genotype of rs2516839 of USF1 (Table 4). There was no association between $-173 \mathrm{G} / \mathrm{C}$ of MIF and lipids profile (data not shown).

\section{Discussion}

Dysfunctions of MIF and USF1 affect cardiovascular disease

\begin{tabular}{|c|c|c|c|}
\hline & Control $(n=455)$ & Case $(n=654)$ & p \\
\hline Age (yr) & $60.4 \pm 10.1$ & $59.80 \pm 9.2$ & 0.056 \\
\hline Males, $\mathrm{n}$ & $262(57.6 \%)$ & $490(74.9 \%)$ & 0.001 \\
\hline Smoking, $\mathrm{n}$ & $146(32.1 \%)$ & $304(46.5 \%)$ & 0.001 \\
\hline Drinking, $\mathrm{n}$ & $105(23.1 \%)$ & $144(22.2 \%)$ & 0.040 \\
\hline $\mathrm{DM}, \mathrm{n}$ & $60(13.2 \%)$ & $96(14.7 \%)$ & 0.606 \\
\hline $\mathrm{HBP}, \mathrm{n}$ & $181(40.0 \%)$ & $460(70.2 \%)$ & 0.001 \\
\hline BMI $\left(k g / m^{2}\right)$ & $23.2 \pm 3.4$ & $24.3 \pm 3.3$ & 0.362 \\
\hline Glucose (mmol/L) & $5.6 \pm 1.23$ & $5.87 \pm 1.31$ & 0.412 \\
\hline $\mathrm{TC}(\mathrm{mmol} / \mathrm{L})$ & $4.5 \pm 0.9$ & $5.2 \pm 1.4$ & 0.197 \\
\hline HDL-C (mmol/L) & $1.8 \pm 0.3$ & $1.2 \pm 0.5$ & 0.032 \\
\hline LDL-C (mmol/L) & $2.6 \pm 0.8$ & $3.4 \pm 1.2$ & 0.083 \\
\hline TG $(\mathrm{mmol} / \mathrm{L})$ & $2.0 \pm 0.9$ & $1.8 \pm 1.4$ & 0.373 \\
\hline apoA (g/L) & $1.2 \pm 0.2$ & $1.1 \pm 0.25$ & 0.475 \\
\hline apoB $(g / L)$ & $0.8 \pm 0.2$ & $0.8 \pm 0.3$ & 0.524 \\
\hline apoE (g/L) & $49.2 \pm 13.5$ & $49.6 \pm 11.4$ & 0.384 \\
\hline $\operatorname{Lp}(a)(g / L)$ & $164.5 \pm 52.3$ & $212.0 \pm 96.0$ & 0.001 \\
\hline
\end{tabular}

Age, BIM, TC, TG, HDL-C, LDL-C and glucose are reported as mean \pm SD. DM, Diabetes mellitus; HBP, hypertension; BMI, body mass index; HDL-C, high density lipoprotein cholesterol; LDL-C, low density lipoprotein cholesterol; TC, total cholesterol; TG, triglyceride; apoA, apolipoprotein A; Lp(a), Lipoprotein(a).

Table 1: Baseline characteristics of cases and controls.

\begin{tabular}{|c|c|c|c|c|c|c|c|c|c|c|c|c|}
\hline \multirow{2}{*}{$\begin{array}{c}\text { SNPs } \\
\text { rs2516839 }\end{array}$} & \multirow{2}{*}{$\begin{array}{l}\text { MAF } \\
0.11\end{array}$} & \multicolumn{3}{|c|}{ Genotype } & \multirow[t]{2}{*}{$p_{1}$} & \multicolumn{2}{|c|}{ Allele } & \multirow[t]{2}{*}{ OR } & \multirow[t]{2}{*}{ OR(95\% Cl) } & \multirow[t]{2}{*}{$p_{2}$} & \multirow[t]{2}{*}{$P(B o n f)$} & \multirow[t]{2}{*}{ P power } \\
\hline & & $\mathrm{G} / \mathrm{G}$ & $\mathrm{G} / \mathrm{A}$ & $\mathrm{A} / \mathrm{A}$ & & G & A & & & & & \\
\hline $\mathrm{CHD}$ & & $220(0.45)$ & $208(0.43)$ & $59(0.12)$ & 0.89 & $648(0.67)$ & $326(0.34)$ & 1.048 & $0.86-1.27$ & 0.64 & 0.87 & 0.1 \\
\hline Non-CHD & & $201(0.47)$ & $179(0.42)$ & $50(0.12)$ & & $581(0.68)$ & $279(0.32)$ & & & & & \\
\hline rs755622 & 0.05 & $\mathrm{C} / \mathrm{C}$ & $\mathrm{C} / \mathrm{G}$ & $\mathrm{G} / \mathrm{G}$ & & C & $\mathrm{G}$ & & & & & \\
\hline $\mathrm{CHD}$ & & $297(0.62)$ & $167(0.34)$ & $21(0.04)$ & 0.65 & $761(0.78)$ & $209(0.22)$ & 0.934 & $0.75-1.17$ & 0.554 & 0.79 & 0.118 \\
\hline Non-CHD & & $275(0.64)$ & $136(0.32)$ & $20(0.04)$ & & $686(0.80)$ & $176(0.20)$ & & & & & \\
\hline
\end{tabular}

Table 2: Allele and genotype frequencies of SNPs between case-control subjects. 


\begin{tabular}{|c|c|c|}
\hline Characteristic & & P value \\
\hline male/ female(numbers) & -0.30 & $<0.01$ \\
\hline Age(years) & -0.13 & $<0.01$ \\
\hline Smoking(cases) & 0.204 & $<0.01$ \\
\hline Hypertension(cases) & 0.176 & $<0.01$ \\
\hline Diabetes(cases) & 0.070 & 0.05 \\
\hline $\mathrm{TC}(\mathrm{mmol} / \mathrm{L})$ & 0.088 & $>0.05$ \\
\hline $\mathrm{TG}(\mathrm{mmol} / \mathrm{L})$ & 0.072 & $>0.05$ \\
\hline $\mathrm{LDL}-\mathrm{C}(\mathrm{mmol} / \mathrm{L})$ & 0.101 & $<0.01$ \\
\hline $\mathrm{HDL}-\mathrm{C}(\mathrm{mmol} / \mathrm{L})$ & -0.169 & $<0.01$ \\
\hline apoA (mgdL) & -0.186 & $<0.01$ \\
\hline apoB $(\mathrm{mgdL})$ & 0.06 & $>0.05$ \\
\hline apoE $(\mathrm{mgdL})$ & 0.05 & $>0.05$ \\
\hline Lp(a) & 0.125 & $<0.01$ \\
\hline rs2516839 genotype & 0.02 & $>0.05$ \\
\hline rs755622 genotype & -0.03 & $>0.05$ \\
\hline
\end{tabular}

TC, total cholesterol; TG, triglyceride; HDL-C, high-density lipoprotein cholesterol LDL-C, low-density lipoprotein cholesterol; apoA, apolipoprotein A; Lp(a), Lipoprotein(a)

Table 3: Phi coefficient of Spearman's correlation analysis.

\begin{tabular}{|c|c|c|c|c|c|c|}
\hline & \multicolumn{2}{|c|}{ Controls } & \multicolumn{2}{c|}{ All subjects } & \\
\hline genotypes & GG & AG+AA & P & GG & AG+AA & P \\
\hline $\begin{array}{c}\text { TC } \\
(\mathrm{mmol} / \mathrm{L})\end{array}$ & $4.17 \pm 0.16$ & $4.54 \pm 0.05$ & $0.027^{*}$ & $4.45 \pm 0.14$ & $4.54 \pm 0.04$ & 0.539 \\
\hline $\begin{array}{c}\mathrm{HDL}-\mathrm{C} \\
(\mathrm{mmol} / \mathrm{L})\end{array}$ & $1.17 \pm 0.12$ & $1.17 \pm 0.02$ & 0.871 & $1.11 \pm 0.03$ & $1.14 \pm 0.02$ & 0.605 \\
\hline $\begin{array}{c}\mathrm{LDL}-\mathrm{C} \\
(\mathrm{mmol} / \mathrm{L})\end{array}$ & $2.26 \pm 0.12$ & $2.61 \pm 0.04$ & $0.008^{*}$ & $2.55 \pm 0.11$ & $2.62 \pm 0.03$ & 0.483 \\
\hline $\begin{array}{c}\mathrm{TG} \\
(\mathrm{mmol} / \mathrm{L})\end{array}$ & $1.14 \pm 0.12$ & $1.21 \pm 0.05$ & 0.668 & $1.24 \pm 0.09$ & $1.29 \pm 0.04$ & 0.636 \\
\hline apoA (g/L) & $1.19 \pm 0.04$ & $1.15 \pm 0.01$ & 0.371 & $1.11 \pm 0.03$ & $1.11 \pm 0.01$ & 0.992 \\
\hline apoB (g/L) & $0.70 \pm 0.03$ & $0.80 \pm 0.01$ & $0.004^{*}$ & $0.80 \pm 0.03$ & $0.82 \pm 0.01$ & 0.461 \\
\hline apoE (g/L) & $46.38 \pm 2.24$ & $49.5 \pm 1.38$ & 0.433 & $48.78 \pm 1.62$ & $49.52 \pm 0.79$ & 0.747 \\
\hline Lp(a) (g/L) & $150.2 \pm 21.30$ & $166.4 \pm 8.8$ & 0.537 & $184.0 \pm 6.3$ & $221.9 \pm 20.8$ & 0.051 \\
\hline
\end{tabular}

TC, total cholesterol; HDL-C, high-density lipoprotein cholesterol; LDL-C, lowdensity lipoprotein cholesterol; TG, triglyceride; apoA, apolipoprotein $A$; $L p(a)$, Lipoprotein(a). *, $\mathrm{P}<0.05$

Table 4: Lipid profiles between the different genotypes of rs2516839 of USF1.

and overall mortality. We investigated the associations of $-173 \mathrm{G} / \mathrm{C}$ (rs755622) of MIF and rs2516839 of USF1 with CAD and lipids disorders based on a Chinese-population. Our results showed no significant association between the polymorphism of rs 2516839 of USF1 with CAD risk, neither -173G/C (rs755622) of MIF. However, the GG genotype carriers of rs2516839 of USF1 showed significantly lower levels of cholesterol, low-density lipoprotein cholesterol and apolipoprotein $\mathrm{B}$.

The polymorphism of $-173 \mathrm{G} / \mathrm{C}$ of MIF is a potentially functional polymorphism to regulate the transcription activity of MIF [6-10]. This variant was reported to be associated with a series of chronic inflammatory diseases in Caucasians, such as rheumatoid arthritis, systemic lupus erythematosus bone loss in postmenopausal women and sepsis. CAD is presently considered as a chronic inflammatory process and no report can be found for $-173 \mathrm{G} / \mathrm{C}$ of MIF with CAD [14-17]. Based on our results, there was no positive association between -173G/ $\mathrm{C}$ of MIF and CAD. This divergence is actually explainable. For one thing, most of the above mentioned positive associations were based on the combination analysis of $-173 \mathrm{G} / \mathrm{C}$ with other polymorphisms, such as $-794 /$ CATT $_{5-8}$ of rs5844572 [14-17]. Indeed, for complex traits such as $\mathrm{CAD}$, haplotype analysis is much more powerful to detect genes with modest effects in comparison to the single-locus test. For another, there is great heterogeneity among different population groups (the genotype frequencies vary between ethnic or geographic populations). For example, the MAF for rs755622 in African American is 0.27, European 0.045, Hispanic 0, Sub-Saharan African 0.167. Our results showed MAF for this SNP is 0.05 , which was consistent with the PUBMED data. Finally, even though the chronic inflammation process has common genes and variants, the complex traits among the inflammatory processes is apparently heterogenetic [18]. Therefore, these points should be definitely taken into consideration in designing association study in the future.

For rs2516839 of USF1, this variant was associated with decreased levels of CHO, LDL-C and apoB in the non-CAD subjects. Our finding was consistent with the study by $\mathrm{Ng} \mathrm{MC}$ et al. [13], who reported that the A allele of rs2516839 was associated with increased risk for metabolic syndrome in a Chinese population. In a most recently report, in females, the serum total, LDL cholesterol and apoB levels were lower in GG genotype of rs 2516839 of USF1 when compared to AA genotype [19]. In another study based on the atherogenic dyslipidemia families, this variant was reported to be associated with increased levels of triglyceride or cholesterol [12]. Actually, in our study, when CAD patients were included, the association of rs2516839 of USF1 with the lipids levels showed negative results. One of the reasons to explain this discrepancy is that in our study, the management of lipids in CAD patients was not included in the analysis, which might constitute a major confounding factor and therefore a definite limitation in our study design.

There were several other limitations in this study. First, our study was performed in a Chinese Han population, and the data should be extrapolated to other population and ethnic groups cautiously. Second, the effects of rs2516839 of USF1 to modify the lipids profile need to be confirmed in a much larger population. Third, further functional experiments are required to analyze the modulation of rs2516839 of USF1 to the lipid accumulation. Fourth, when we do the association analysis for rs755622 of MFI, the haplotype, such as -794/CATT ${ }_{5-8}$ of rs5844572, needed to be considered.

In summary, our findings showed that the rs2516839 of USF1 and $-173 \mathrm{G} / \mathrm{C}$ (rs755622) of MIF do not contribute to CAD risk. Nevertheless, the rs2516839 of USF1 might have a protection for the dyslipidemia disorders.

\section{Acknowledgement}

This work was supported by the General Program (Key Program, Major Research Plan) of National Natural Science Foundation of China (81230007) National Basic Research Program of China (2011CB503905), and National Natural Science Foundation of China (81200147, 81200185).

\section{References}

1. Santulli G (2012) Coronary heart disease risk factors and mortality. JAMA 307 1137.

2. Santulli G (2013) Epidemiology of Cardiovascular Disease in the 21 st Century: Updated Numbers and Updated Facts. Journal of Cardiovascular Dsease 1: 3

3. Alam A, Halder S, Thulasiram HV, Kumar R, Goyal M, et al. (2012) Novel antiinflammatory activity of epoxyazadiradione against macrophage migration inhibitory factor: inhibition of tautomerase and pro-inflammatory activities of macrophage migration inhibitory factor. J Biol Chem 287: 24844-24861.

4. Miller EJ, Li J, Leng L, McDonald C, Atsumi T, et al. (2008) Macrophage migration inhibitory factor stimulates AMP-activated protein kinase in the ischaemic heart. Nature 451: 578-582

5. Donn R, Alourfi Z, De Benedetti F, Meazza C, Zeggini E, et al. (2002) Mutation screening of the macrophage migration inhibitory factor gene: positive 
Citation: Wu C, Gong Y, Zhu X, Sun A, Yuan J, et al. (2014) The Polymorphisms rs2516839 of USF1 and -173G/C of MIF were not Associated with Coronary Artery Disease but Dyslipidemia in a Chinese Population. J Cardiovasc Dis Diagn 2: 177. doi:10.4172/2329-9517.1000177

association of a functional polymorphism of macrophage migration inhibitory factor with juvenile idiopathic arthritis. Arthritis Rheum 46: 2402-2409.

6. Martínez A, Orozco G, Varadé J, Sánchez López M, Pascual D, et al. (2007) Macrophage migration inhibitory factor gene: influence on rheumatoid arthritis susceptibility. Hum Immunol 68: 744-747.

7. Wu J, Chen F, Zhang X, Li Y, Ma H, et al. (2009) Association of MIF promoter polymorphisms with psoriasis in a Han population in northeastern China. $J$ Dermatol Sci 53: 212-215.

8. Meyer-Siegler KL, Vera PL, Iczkowski KA, Bifulco C, Lee A, et al. (2007) Macrophage migration inhibitory factor (MIF) gene polymorphisms are associated with increased prostate cancer incidence. Genes Immun 8: 646652

9. Xue Y, Xu H, Rong L, Lu Q, Li J, et al. (2010) The MIF -173G/C polymorphism and risk of childhood acute lymphoblastic leukemia in a Chinese population. Leuk Res 34: 1282-1286.

10. Sakaue S, Ishimaru S, Hizawa N, Ohtsuka Y, Tsujino I, et al. (2006) Promoter polymorphism in the macrophage migration inhibitory factor gene is associated with obesity. Int J Obes (Lond) 30: 238-242.

11. Naukkarinen J, Nilsson E, Koistinen HA, Soderlund S, Lyssenko V (2009) Functional variant disrupts insulin induction of USF1: mechanism for USF1associated dyslipidemias. Circulation Cardiovascular genetics 2: 522-529.

12. Laurila PP, Naukkarinen J, Kristiansson K, Ripatti S, Kauttu T, et al. (2010) Genetic association and interaction analysis of USF1 and APOA5 on lipid levels and atherosclerosis. Arteriosclerosis, thrombosis, and vascular biology 30: 346-352.
13. Ng MC, Miyake K, So WY, Poon EW, Lam VK, et al. (2005) The linkage and association of the gene encoding upstream stimulatory factor 1 with type 2 diabetes and metabolic syndrome in the Chinese population. Diabetologia 48 2018-2024.

14. Baugh JA, Chitnis S, Donnelly SC, Monteiro J, Lin X, et al. (2002) A functiona promoter polymorphism in the macrophage migration inhibitory factor (MIF) gene associated with disease severity in rheumatoid arthritis. Genes Immun 3: 170-176.

15. Sreih A, Ezzeddine R, Leng L, LaChance A, Yu G, et al. (2011) Dual effect of the macrophage migration inhibitory factor gene on the development and severity of human systemic lupus erythematosus. Arthritis Rheum 63: 3942 3951.

16. Swanberg M, McGuigan F, Ivaska KK, Gerdhem P, Lerner UH, et al. (2010) Polymorphisms in the macrophage migration inhibitory factor gene and bone loss in postmenopausal women. Bone 47: 424-429.

17. Lehmann LE, Book M, Hartmann W, Weber SU, Schewe JC, et al. (2009) A MIF haplotype is associated with the outcome of patients with severe sepsis: a case control study. J Transl Med 7: 100

18. Wellcome Trust Case Control C (2007) Epidemiology of Cardiovascular Disease in the 21 st Century: Updated Numbers and Updated Facts. Nature 447: 661-678.

19. Fan YM, Hernesniemi J, Oksala N, Levula M, Raitoharju E, et al. (2014) Upstream Transcription Factor 1 (USF1) allelic variants regulate lipoprotein metabolism in women and USF1 expression in atherosclerotic plaque. Scientific reports 4: 4650 . 